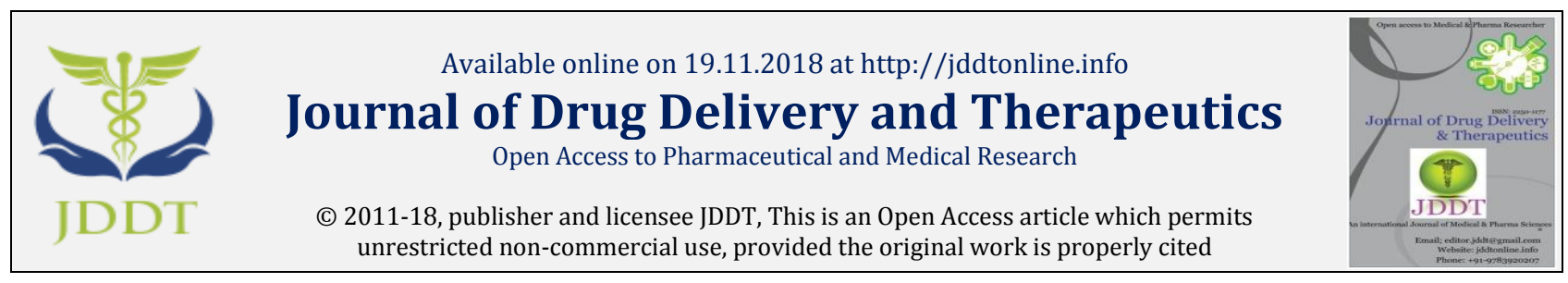

Open $\odot$ Access

Review Article

\title{
A comprehensive review on pharmaceutical mini tablets
}

\author{
Priyanka, Kapil Kumar*, Deepak Teotia \\ Global Institute of Pharmaceutical Education and Research, Kashipur, Uttrakhand, India
}

\begin{abstract}
Mini-tablets represent a new trend in solid dosage form design, with the main goal of overcoming some therapeutic obstacles. Mini tablets are multiple unit dosage forms and are advantageous than pellets or any other oral dosage forms as they are easy to manufacture and stability problems are less. Offering some therapeutic benefits such as dose flexibility and combined release patterns. They do not require any solvent for their production and also local irritation can be avoided by the use of mini tablet Mini tablet offer several advantages like they can be manufactured relatively easily, They are not require less coating materials and also there is a great flexibility during their formulation development. Mini tablet are more acceptable in children and elderly people as they are easy to swallow. The objective of controlled drug delivery systems is to reduce the frequency of the dosing and to increase the effectiveness of the drug by localization.
\end{abstract}

Keywords: Mini-tablets, solid dosage form, oral dosage forms.

Article Info: Received 05 Oct, 2018; Review Completed 06 Nov 2018; Accepted 14 Nov 2018; Available online 19 Nov 2018

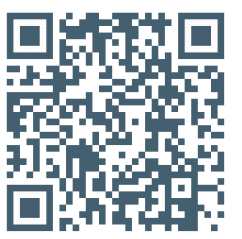

\section{Cite this article as:}

Priyanka, Kumar K, Teotia D, A comprehensive review on pharmaceutical mini tablets, Journal of Drug Delivery and Therapeutics. 2018; 8(6):382-390 DOI: http://dx.doi.org/10.22270/jddt.v8i6.2060

\section{*Address for Correspondence:}

Kapil Kumar, Global Institute of Pharmaceutical Education and Research, Kashipur, Uttrakhand, India

\section{INTRODUCTION}

Tablets are the most widely used solid dosage form and there is always scope for improving the limitations of tablets like, difficulty in swallowing and delay in the onset of Action 1. Mini- tablets are novel multiple unit solid dosage form which are in the size equal to or smaller than $3.0 \mathrm{~mm}$ in Diameter ${ }^{2}$ Over a decade now Fastdisintegrating tablets (FDTs), have gained enormous popularity for better patient compliance and Acceptance 3 . FDTs ease of administration without water and provide the fast onset of action is also the reason for their gaining popularity in the general Population ${ }^{4}$. Also, FDTs have the proven potential in increasing the bioavailability of low aqueous soluble drug through fast disintegration and enhanced dissolution rate 5.Mini- tablets are novel multiple unit solid dosage form which are in the size equal to or smaller than $3.0 \mathrm{~mm}$ in diameter 6 .Oral administration of medicines has an advantage for patient's compliance. Most of the solid dosage forms administered orally is tablets. Tablets have many advantages over other dosage forms, such as ease of transportation, application and production, high patient compliance, accurate dosing, control of drug release and stability. However, the desired release profile, therapeutic effect or ease of use in pediatrics or geriatrics, difficulty in swallowing may not be achieved by conventional tablets. Drug delivery system ensures reaching the effect area of the administered drug and sufficient concentration of the drug at the site of action.
These factors, such as repetitive dosing, unpredictable absorption and undesirable toxicity lead to the development of controlled drug delivery system. The main aim in designing sustained or controlled drug delivery systems is to reduce the frequency of the dosing and to increase the effectiveness of the drug by localization at the specific site of action.

Mini-tablets are tablets with a diameter $\leq 3 \mathrm{~mm}$ produced on conventional tablet presses equipped with multiple tooling. This production is similar to the production of standard tablets but requires excellent powder flow due to the small dies, exact control of process parameters and special caution during tablet press assembly in order to avoid tool damage. Mini-tablets (coated or uncoated and single or multiple-unit systems) are mainly developed as patient-friendly systems for pediatric and geriatric patients and also for personalized medicine because they offer improved swallowing and flexible dosing, combining various release kinetics, doses and active compounds in only one system. Mini-tablets may also be successfully used as multiple-unit modified release systems (extended release, delayed-colon release, pulsatile and bi-modal release and gastro retentive systems) providing improved drug bioavailability compared with single-unit systems.

Oral controlled release drug delivery systems can be classified in two categories: 
- Single unit dosage forms (SUDFs), like tablets or capsules, and

- Multiple unit dosage forms (MUDFs), like granules, pellets or mini-tablets.

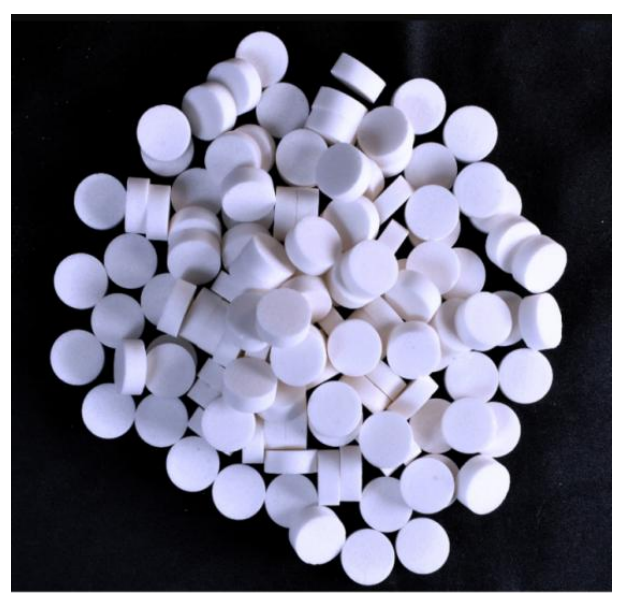

Figure 1: Mini tablets

Mini tablet is with diameter of $3-6 \mathrm{~mm}$. Majority of the drugs absorption is more in upper part of small intestine (duodenum), for a drug to reach the small intestine it had to pass through stomach ${ }^{6}$. So, drug absorption depends on gastric empting time. If the gastric emptying is too fast drug may not absorb to required level or if it too slows it may get mix-up with gastric contents and may adsorb to food which gives unintended effects. These effects are more in case of single unit dosage forms because of their size but in case of mini tablets will not depend on gastric emptying and easily get passed through pylorus. So mini tablets are beneficial over the normal size tablets to reduce intra and inter subject variability. Mini tablets will give reproducible plasma drug concentrations. Plasma drug concentration is directly proportional to the absorption ${ }^{7}$. Absorption is more and even with mini tablets as they are distributed all over the surface which is not in case of single unit dosage forms. So by formulating multiple unit dosage forms like mini tablets better plasma drug profiles can be obtained. Mini tablets can be easily divided and administered without loss of activity. Elderly and pediatrics patients who sometimes chew the tablets which releases drug all at once and may cause toxicity in case of normal tablet but in case of Mini tablets, they can be chewed as here each mini depot in the formulation act individually dose dumping may not occur. For local irritating drugs, mini tablet formulation decreases the irritation effect than that of single unit formulations ${ }^{7}$

\section{Constituents of Mini-Tablets}

Different mini-tablets can be formulated and designed individually, incorporated into a capsule to release the drug at different sites and at different rates. Different combinations of mini-tablets include immediate release, delayed release, and/or controlled release formulations. Also, combining different mini-tablets together, incompatible drugs can be administered. This, as a result, improves overall therapeutic outcome, and also concurrent diseases can be treated effectively ${ }^{8}$.

\section{Release profile}

Due to increased surface in relation to volume, the drug can be released more efficiently in case of mini-tablets. By applying uniform layer of a retarding film coat, the release rate of the drug can be controlled with greater certainty. Also, mini-tablets that are formulated using different concentrations of HPMC $\mathrm{K} 100 \mathrm{M}$, provides a prolonged drug release rates. The drug contained in the mini-tablets gets released at different rates, depending upon composition of mini tablets. Based on the release kinetic parameters calculated, it can be concluded that minitablets containing HPMC K100M are particularly suitable to release the drug over hours of time periods ${ }^{9}$.

By combining different doses of mini tablets, it is possible to achieve various releases with one formulation. Due to significant smaller dimensions of the mini tablets, when compared to normal tablets, they pass through the stomach at more even rate. As a result, the concentration of the drug in the blood can be easily reproduced.

\section{Advantages of Mini Tablets:}

- Mini Tablets can be manufactured relatively easily.

- They offer high drug loading, a wide range of release rate designs, and fine tuning of these release rates ${ }^{10}$.

- They have excellent size uniformity, regular shape and smooth surface.

- They offer high degree of dispersion in the GI tract, thus minimizing the risks of high local drug concentrations.

- They have less risk of dose dumping11.

- They have less risk of dose dumping, less inter- and intra- subject variability, high degree of dispersion in the digestive tract thus minimizing the risks of high local drug concentrations.

- They can be produced in a reproducible and continuous way.

- They combine the advantages of MUDFs with the established manufacturing techniquesin tableting and have fewer constraints compared to extrusion/spheronization technique ${ }^{12}$.

- They offer high drug loading, a wide range of release rate patterns, and also fine tuning of these release rates.

- Mini-tablets also offer an alternative for pellets because of their relative ease of manufacturing and because dosage forms of equal dimensions and weight with smooth regular surface are produced in a reproducible and continuous way.

- They offer a substrate which is easy to coat with polymeric membranes for modified release purposes.

- Mini tablets have less inter and intra- subject variability.

- Mini tablets are easy to manufacture compared to pellets as they have equal dimensions, weight with smooth regular surface ${ }^{13}$.

- Mini tablets are good coating substrates as they have excellent size uniformity, regular shape and a smooth surface.

Unlike pellets, mini tablets do not require any solvents for its production; as a result problems with stability can be avoided ${ }^{9,10 .}$

\section{Advantages of mini tablets over pellets}

- Mini tablets do not require any solvents for its 
production; as a result problems with stability can be avoided ${ }^{14}$.

- Pellets are small bead like structures, usually with medium to high uniformity and are usually filled into capsules (Figure 2) or compressed into tablets.

- Technically demanding process like fluid bed granulation, extrusion or spheronization are required for the production of pellets. Whereas, mini tablets can be manufactured via simple tabletting procedures ${ }^{15}$.

As mini-tablets have well designed size, shape, smooth surface, low degree of porosity and high mechanical strength, they are easy to coat than pellets, which usually have an uneven surface and are very porous. Hence, tablets with defined size, shape and surface can be easily produced with good batch to batch uniformity.

\section{Possibilities of Formulating Mini-Tablet Dosage Forms}

1. Compressed mini-tablets

2. Encapsulated Coated mini-tablets

3. Compressed mini-tablets presented as a biphasic drug delivery system

\section{Compressed mini tablet}

In order to avoid the cost of hard gelatin capsules, mini tablets can be formulated as tablet. Uniform sizes, smooth shapes, smooth surfaces, low porosity and high mechanical resistance make them more uniform and reproducible tablets than pellets and granules ${ }^{16}$. Depending on the properties of the external phase that provides the filling of the cavity (hydrophobic / hydrophilic polymer matrix used and the number of mini tablets), release profile can be changed. Biphasic drug delivery systems are developed using different release characteristics. In these systems, one phase initiates the rapid action by providing the immediate release while the other phase releases the long-term effect, ensuring continuity of efficacy and eliminating the need for recurrent doses of the drug $13,14,15$.

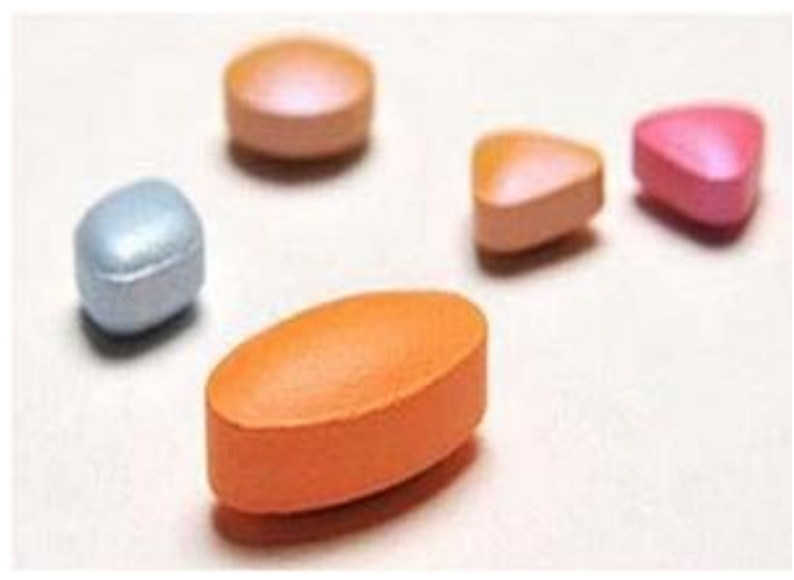

Figure 2: Compressed Mini-tablets

\section{Encapsulated coated mini-tablets systems}

Coated oral sustained-release forms of drugs are widely used to improve drug tolerance or to yield a dosing regimen that is easier to manage for patients. However, little published information is available on sustainedrelease systems using coated mini-tablets ${ }^{17}$.

In particular, it has proven challenging to develop one dosage form with sustained and immediate-release properties. A multifunctional and multiple unit system, which contains versatile mini-tablets in a hard gelatin or HPMC capsule, can be developed by preparing Rapidrelease Mini-Tablets (RMTs), Sustained-release MiniTablets (SMTs), Pulsatile Mini Tablets (PMTs), and Delayed-onset Sustained-release Mini-Tablets (DSMTs), each with various lag times of release. Based on the combinations of mini-tablets, multiplied pulsatile drug delivery system (DDS), site-specific DDS, slow/quick DDS, quick/slow DDS, and zero order DDS could be obtained. Inclusion of RMTs permits the development of rapid-acting encapsulated dosage forms with optimal pharmacokinetic profiles for fast action. The size of the tablet can be reduced such that it could be enclosed in a capsule, then deploy tablets with different release properties within the one capsule. Several mini-tablets can be placed into each HPMC capsule, which later disintegrates and releases these subunits. Because several mini tablets can be placed into each capsule, tablets with different combination of drugs, dose and drug-release profiles can be included ${ }^{18}$.

\section{Compressed mini-tablets systems are presented as a biphasic delivery system:}

Biphasic delivery systems are designed to release a drug at two different rates or in two different periods of time: they are either quick/slow or slow/quick. A quick/slow release system provides an initial burst of drug release followed by a constant rate (ideally) of release over a defined period of time and in slow/quick release system provides release vice versa. Biphasic release system is used primarily when maximum relief needs to be achieved quickly, and it is followed by a sustained release phase to avoid repeated administration. Suitable candidate drugs for this type of administration include non-steroidal anti-inflammatory drugs (NSAIDs) antihypertensive, antihistaminic, and antiallergic agents ${ }^{19}$. Generally, conventional controlled dosage forms delay the release of therapeutic systemic levels and do not provide a rapid onset of action. While immediate release granules give fast release to provide rapid onset of action, but fails to provide longer duration of action. A relatively constant plasma level of a drug is often preferred to maintain the drug concentration within the therapeutic window. However, it is difficult to achieve, especially for once-daily dosage forms, partly because the environment for drug diffusion and/or absorption varies along the gastrointestinal (GI) tract. On the basis of these considerations, we have proposed a new oral delivery device, in the form of a double-component tablet and granules, in which the one portion is formulated to obtain a prompt release of the drug, with the aim of reaching a high serum concentration in a short period of time. The second portion is a sustain release matrix, which is designed to maintain an effective plasma level for a prolonged period of time ${ }^{20}$. This concept can be used to produce a biphasic delivery system combining a fast release together with the slow release period of the drug, provided that the excipients powder that fills The void spaces between the mini-tablets incorporate a part of the total drug dose. This system can produce a rapid rise in the plasmatic concentrations for some drugs (such as analgesic, antiinflammatory, anti hypertensive and antihistaminic agents) that are requested to promptly exercise the therapeutic effect, followed by an extended release phase in order to avoid repeated administrations ${ }^{17}$. 


\section{Types of Mini tablets}

Enteric coating is a barrier applied to oral medication that controls the location in the digestive system where it is absorbed. Most enteric coatings work by presenting a surface that is stable at the highly acidic $\mathrm{pH}$ found in the stomach, but breaks down rapidly at a less acidic (relatively more basic) pH. For example, they will not dissolve in the acidic juices of the stomach $(\mathrm{pH} \sim 3)$, but they will in the alkaline (pH 7-9) environment present in the small intestine. Materials used for enteric coatings include fatty acids, waxes, shellac, plastics, and plant fibres. Drugs that have an irritant effect on the stomach, such as aspirin, can be coated with a substance that will dissolve only in the small intestine. Likewise, certain groups of azoles (esomeprazole, omeprazole, pan and all grouped azoles) are acid-activated. For such types of drugs, enteric coating added to the formulation tends to avoid activation in the mouth and oesophagus. Recently, some companies have begun to utilize enteric coatings on fish oil (omega-3 fatty acids) supplements. The coating prevents the fish oil capsules from being digested in the stomach, which has been known to cause a fishy reflux (fish burps). Sometimes the abbreviation "EC" is added beside the name of the drug to indicate that it has an enteric coating 21 .

Mini tablets can be classified based on the target site, method of manufacturing, patient needs as follows:

\section{Pediatric mini tablets \\ 2. Oral disintegrating mini tablets \\ 3. Gastro retentive min tablets \\ 4. Bio-adhesive mini tablets \\ 5. Biphasic mini tablets 20,21.}

\section{Pediatric mini tablets}

Syrups, tablets and capsules are commonly used dosage forms for children. Syrups are liquid dosage forms which are simple to administer and dose can be easily altered to the patient needs on the other side disadvantages with these liquids dosage forms are chemical, physical, and microbial instability, taste issues, lack of controlled release and formulation problems. In case of tablets as they are big in size difficulty in swallowing and dose adjustment is difficult. Some time we have to break the tablets and administer which causes loss of activity of the tablets. Patient compliance is another issue with the conventional dosage forms. To overcome all the above issues formulating mini tablets can result in good patient acceptance. Mini tablets are easily accepted by children than other dosage forms like tablets, syrups, and capsules etc 22 .

\section{pH responsive mini tablets}

The $\mathrm{pH}$ of human Gastro Intestinal Tract varies greatly (Stomach 1.5-3.0, upper part of small intestine Duodenum 4.0-5.0, lower parts of SI jejunum and ileum 6.5-7.5, and colon 5.6-6.9). $\mathrm{pH}$ responsive drug release is required when absorption of drug is more at a particular site this can be achieved by coating with $\mathrm{pH}$ responsive release polymers like Eudragits. Generally coating is done to granules and then they are filled into capsules to achieve the required release at required tablets can be used as an alternative to pellets. M A Hadi et al. formulated $\mathrm{pH}$ responsive mini tablets for ileo colonic drug delivery of naproxen which is used for the treatment of rheumatoid arthritis. Eudragit L100 and Eudragit S100 are used as pH responsive polymers to get the required release ${ }^{23}$.

\section{Oral disintegrating mini tablets}

Oral dispersible tablets (ODTs) are the novel dosage form which rapidly disintegrates in the mouth (1-3 min) without chewing upon oral administration and without the need of water, unlike other conventional oral solid dosage form.

Oral Dispersible Tablets (ODTs) are also known as "fast dissolve", "rapidly disintegrating", "quick-dissolve", "crunch-melt", "bite-dispersible", "mouth-dissolve", and "orodispersible" tablets. Oral dispersible mini tablets (ODMTs) are more suitable for paediatric patients because of their small size, pleasant mouth feel and fast disintegration in mouth. The ODT should have the following characters they should disintegrate in the mouth without additional water. The disintegrated tablet should become a soft paste or liquid suspension, which can provide good mouth feel and smooth swallowing. Because ODTs dissolve or disintegrate in the patient's mouth, the drug will be partially dissolved in close proximity to the taste buds. A pleasant taste inside the mouth becomes critical for patient acceptance. Unless the drug is tasteless or does not have an undesirable taste, taste-masking techniques should be used. The taste-masking technology should also be compatible with ODT formulations ${ }^{22}$

\section{Gastro retentive mini tablets or Floating mini tablets}

Gastro retentive mini tablets are intended to release the drug in stomach for prolonged time. Generally for tablets to float on the GI fluids content we formulate tablets by using gas generating agents in them. These tablets when come in contact with food generate $\mathrm{CO}_{2}$ and the generated gas is trapped in swellable hydrocolloid which makes the tablet to float and retain in stomach. In normal single unit tablets drug loading is low as the polymer used for floating in high. In mini tablets we can use coating with sodium bicarbonate or calcium carbonate (gas generating agents), eudragits coating in place of swellable polymers used in formulation to increase the drug loading. Fluid bed processor can be used for coating of mini tablets. Goole et al developed sustained release floating mini tablets of levodopa. Here they used $3 \mathrm{~mm}$ mini tablets core formulated with gas generating agent and coated the core with eduragit RL30 D to get the required release 23 .

\section{Bioadhesive Vaginal Mini Tablets}

The various available dosage forms for vaginal drug delivery are creams, gels, ointments and tablets. The problems with these are leakage, messy, less patient compliance and less retention time. Nano pharmaceuticals can be used but the problem associated with them is low residence time as they are liquid in nature. To overcome the above problems we can use bio adhesive polymers. Bio adhesive polymers or hydrophilic polymers are readily soluble and adhesive on exposure to moisture and will rapidly cohere to surfaces as they have high viscosity at low concentrations. Solid dosage forms have high dose accuracy than semisolids systems. The problem in solid dosage forms is vaginal disintegration is slow and they are rapidly cleared due to gravity and self-cleansing action of vagina. Bio adhesive polymers can be used to overcome this but in large size tablets loss is reported 5 .

Bio adhesive mini tablets can be used for vaginal drug delivery to deliver drug accurately and for long period of time. In mini tablets dose is divided into multiple units which will spread evenly in vaginal cavity with improved coverage in vaginal epithelium. Bio adhesive Mini tablets act by swelling and forming micro gels and releasing drug in controlled release manner and there by maximum bioavailability can be achieved. 
Marianne Hiorth et al were Prepared Bioadhesive mini tablets of hexyl ammonium hydro chlorodium (HAL), which are used for the photodynamic therapy of cervical cancer. Thermo gel of HAL is already there but HAL is unstable in moist environment, to prevent or to eliminate $t$ he stability issues bio adhesive mini tablets were prepared by direct compression 7 . Mini tablets prepared with HPMC and HPC have shown adequate mechanical and bio adhesive properties. Vaginal $\mathrm{pH}$ varies from women to women of different ages. To withstand those pHconditions bio adhesive vaginal mini tablets are to be designed by using non-ionic cellulose ethers with Bioadhesive property 24.

\section{Methods of manufacturing mini tablets:}

Some of the methods that can be used for the manufacturing of mini tablets are

1. Direct compression

2. Wet granulation

3. Dry granulation

4. Melt- extrusion

\section{Direct compression technique}

Direct compression is the process by which tablets are compressed directly from powder blends containing API and excipients directly compressed the powder blend into biconvex mini tablet. Excipients of direct compression grade are used here to get the required hardness. Stability problems are less compared to that of tablets prepared by wet granulation 21 .

\section{Wet granulation}

Wet granulation involves the use of binder solution to form granules which then compressed in compression machine to get mini tablets. Polyvinyl pyrrolidone of different grades is generally used as a binding agent.

\section{Dry granulation technique}

Dry granulation is rational technique of choice for the manufacture of tablets containing thermo labile and moisture-sensitive drugs. This technique employs processing equipment known as roller compactor or chilsonator. This machine compress as premixed powders between two counter rotating rollers under extreme pressure. The resultant material is in the form of a brittle ribbon, sheet, or piece-depending on the configuration of the roller. The compressed material is reduced to the proper size to form granules that are mixed with other inactive excipients and finally compressed on a rotary compression machine ${ }^{15}$.

\section{Melt-Extrusion technique}

In melt-extrusion technique, the powder (API+ excipients) were premixed this premixed powder is then transferred to melt-extruder. In melt-extruder parameters like screw speed, feed rate and temperature are set in the range of melting point range of material. After the process the extrudates are then milled and sieved. The obtained granules are then compressed to mini tablets using compression machine 25 .

Encapsulated mini-tablets system usually comprises immediate-release mini-tablets (IRMT) and sustained release mini-tablets (SRMT) in a capsule made from HPMC, a water-soluble polymer. HPMC capsule which contains the mini-tablets later disintegrates and releases these subunits into the system. As several mini-tablets can be placed in each capsule, tablets with different dose, content and release characteristics can be included ${ }^{18}$.

Inclusion of IRMT permits the development of rapid acting dosage forms for fast action. Encapsulated minitablets systems can be designed to yield various sustained release drug profiles by combining different types, quantities and combinations of mini-tablets, thereby improving patient compliance.

Mini-tablets are usually coated with enteric coating polymers in fluid bed coater or in modified coating pans ${ }^{17}$.

Enteric coating is a polymer barrier, which when applied to a drug protects it from the acidic $\mathrm{pH}$ of the stomach, and releases the drug in the alkaline environment of the small intestine. That is, they will not get dissolved in the acidic juices of the stomach, but breaks down in the alkaline environment of the small intestine. Materials used for enteric coatings mostly include fatty acids, waxes, phthalates, shellac, plastics, and plant fibres ${ }^{21}$.

Drugs that cause irritation to gastric mucosa or inactivated in the stomach, can be coated with a substance that will dissolve only in the small intestine. Abbreviation "EC" along with the name of the drugindicates that the drug has an enteric coating 26 .

\section{Formulation of mini-tablet-in-capsule systems}

The formulation process of mini-tablet-in-capsule systems canbe divided into three important steps:

- The formulation/production of mini-tablets,

- Coating of these mini-tablets with appropriate coating polymer, Filling of coated mini-tablets into hard gelatin or HPMC capsules (Mini-tablets-in-capsule systems) ${ }^{17}$.

Preparation of mini-tablets-in capsule system and granules-mini-tablets-in-capsule systems: Formula used for the calculation of immediate-release dose

The pharmacokinetic parameters of drug were utilized for the calculation of theoretical drug release profile for coated mini-tablet-in-capsule system. The immediate-release part of drug was calculated using the following equation.

$\mathrm{DL}=\mathrm{C}_{\max } \mathrm{V}_{\mathrm{d}}$

Where $C_{\max }$ is maximum plasma concentration, and $V_{d}$ is volume of distribution.

\section{Preparation of immediate release component (Granules)}

Calculated amount of immediate-release dose drug and other suitable excipients [Microcrystalline cellulose (Avicel $\mathrm{PH}$ 102)] were used because of its good compaction and disintegration properties. Any suitable superdisintegrants was used to obtain an immediate release of the drug. The granules were prepared by wet granulation method 22 .

\section{Preparation of immediate-release coated mini-tablet} (IRCMT)

The IRCMT was prepared using the wet granulation method.

\section{Preparation of sustained-release coated mini-tablet (SRCMT)}

The SRCMT was prepared using the same method as used for preparing the IRCMT. However, the SRCMT did not contain the any superdisintegrants. A coating suspension was prepared from HPMC $(5 \mathrm{cps} / 15 \mathrm{cps})$, ethyl cellulose, magnesium stearates, ethyl alcohol and water. Magnesium 
stearate was used in the coating preparation to minimize friction between the surfaces of mini- tablets, the minitablets-filling system and the HPMC capsules ${ }^{18}$.

\section{Preparation of coated mini-tablet-in-capsule system}

To prepare the CMTICS, two IRCMT and three SRCMT were placed in each HPMC capsule (size1). Both similar/different ratios of SRCMT were placed in each HPMC capsule to achieve various sustained release profiles of the CMTICS 27,28 .

\section{Mini tablets can be administered by following methods}

- Directly administered as single units.

- Filled in hard gelatine capsules.

- Use of automatic Dose dispensing device ${ }^{29}$.

\section{Directly administered as single units}

Mini tablets can be directly administered as such. Required dose can be easily taken and these are packed in bottles. Sometime compressed mini tablets are again compressed to get tablets of normal size.

\section{Filled in hard gelatin capsules}

As it is difficult to handle the mini tablets these are usually filled in hard gelatin capsules and then administered ${ }^{30}$.

\section{Automatic dose dispensing device}

Dose is decided on the basis of patient population average dose individualization is important as administration of right drug in wrong dose will result in adverse effects of decreased efficiency. Generally tablets are most commonly used but limited strengths available for administration. Dividing tablets for getting required dose or combining different strengths will not give the desired therapeutic effect so an automatic dose dispensing device can used to dispense tablets of required dose ${ }^{31}$.

\section{Disintegration test}

a. Hard gelatin capsules: Disintegration time shall not be more than $30 \mathrm{~min}$.

b. Soft gelatin capsules: Disintegration time shall not be more than $60 \mathrm{~min}$.

c. Enteric capsule: In Acidic media; shall not disintegration 2 hrs and in alkaline medium capsules shall disintegrate within $30 \mathrm{~min}^{28}$.

\section{F) In-vitro drug release}

- Mini-tablets were subjected to in-vitro drug release studies in simulated gastric and intestinal fluids to assess their ability in providing the desired controlled drug delivery ${ }^{29}$.

- Drug release studies3,54, 55 were carried out using USP dissolution test apparatus I at $100 \mathrm{rpm}, 37 \pm 0.5^{\circ} \mathrm{C}$, and $\mathrm{pH} 1.2$ buffer $(900 \mathrm{ml})$ (i.e. $0.1 \mathrm{~N} \mathrm{HCl})$ for 2 hours, since the average gastric emptying time is about 2 hours. The dissolution medium was replaced with $\mathrm{pH}$ 6.8 phosphate buffer $(900 \mathrm{ml})$ and experiment continued for another 10 hours. At different time intervals, $5 \mathrm{ml}$ of the samples were withdrawn and replaced with $5 \mathrm{ml}$ of drug-free dissolution medium $^{30}$.

- The samples withdrawn were analyzed by UV spectrophotometer using multi component mode of analysis at required wave length.

\section{Preformulation studies mini-tablets}

Preformulation study relates to pharmaceutical and analytical investigation carried out proceeding and supporting formulation development efforts of the dosage form of the drug substance. It gives information needed to define the nature of the drug substance and provide frame work for the drug combination with pharmaceutical excipients in the dosage form. Hence, the following preformulation studies were performed on the obtained sample of drug ${ }^{17}$.

1. Angle of repose

2. Bulk density and Tapped density

3. Carr's index

4. Hauser's ratio

\section{Angle of repose}

The angle of repose is determined by fixed funnel and free standing cone methods employ a funnel that is secured with its tip at a given height, $h$, which was kept $2 \mathrm{~cm}$ above graph paper that is placed on a flat horizontal surface ${ }^{15}$.

\section{Bulk density and tapped bulk density}

Bulk density and tapped bulk density was determined. A quantity of $2 \mathrm{gm}$ of granules from each formula, previously light Shaken for the break of any agglomerates formed, was introduced into the $10 \mathrm{ml}$ of measuring cylinder. After the initial volume was observed, the cylinder was allowed to fall down its own weight from the hard surface from a height of $2.5 \mathrm{~cm}$ at $2 \mathrm{sec}$ Intervals. The tapping was continued until no further change in the volume was noted LBD and TBD were calculated using the following formulas: LBD: Weight of the powder/volume of the packing. ${ }^{13}$ TBD: Weight of the powder/Tapped volume of the packing ${ }^{15}$.

\section{Compressibility index}

The compressibility index of the granules was determined by Carr's Compressibility index. Carr's index (\%) = [(TBDLBD) * 100] / TBD

Where, LBD: Weight of the powder/volume of the packing. TBD: Weight of the powder/Tapped volume of the packing [30].

\section{Hausner's ratio}

Hausner's ratio can be determined by the following equation, Hausner's ratio = TBD / LBD Where, TBD Tapped bulk densities \& LBD- Loose bulk densities ${ }^{31}$.

1. $<1.25-$ Good flow $=20 \%$ Carr

2. 1.25 - Poor flow $=33 \%$ Carr's

3. Even Hausner's ratio indicates the flow properties of the powder blend and is measured by the ratio of tapped density to bulk density.

Hausner's ratio=Tapped density/Bulk density

\section{Drug excipients Compatibility study}

1. Compatibility of the drug with excipients was determined by FT-IR spectral analysis DSC thermal analysis, this study was carried out to detect any changes on chemical constitution of the drug after combined it with the excipients. The samples were taken for FT-IR and DSC studies ${ }^{31 .}$

\section{a) FTIR studies}

IR spectra for pure drug and best mini-tablets formulations 
were recorded in a Fourier transform infrared (FTIR) spectrophotometer (Shimadzu Corporation 8600, Japan) with $\mathrm{KBr}$ pellets ${ }^{32}$.

\section{b) DSC studies}

DSC studies were carried out for pure drug and best minitablets formulations. DSC scan of about $5 \mathrm{mg}$ accurately weighed montelukast and optimized formulations were performed by using an automatic thermal analyzer system (DSC60 Shimadzu Corporation, Japan). Sealed and perforated aluminium pans were used in the experiments for all the samples. Temperature calibrations were performed using indium as standard. An empty pan sealed in the same way as for the sample was used as a reference. The entire samples were run at a scanning rate of $10^{\circ}$ $\mathrm{C} / \mathrm{min}$ from $50-300^{\circ} \mathrm{C}^{32}$.

\section{EVALUATION OF MINI-TABLETS}

\section{Weight Variation Test}

For this test, 20 tablets are selected randomly from the batch and the individual weight of each tablet is noted.

From this, the average weight is calculated. According to USP, none of the individual tablet weight should be less than $90 \%$ and more than $110 \%$ of the average weight. The specification of weight variation is $10 \% 33$.

\section{Hardness}

The hardness of the Mini tablet is determined using Pfizer hardness tester and expressed in $\mathrm{kg} / \mathrm{cm} 2$. Six tablets were randomly picked from each formulation and the mean and standard deviation values were calculated $33-37$

\section{Thickness}

Thickness of the Mini tablet is measured using a digital calipers and screw gauge. It is expressed in terms of $\mathrm{mm}^{38}$.

\section{Friability (F)}

Friability test of Mini tablets is conducted using Roche friabilator. For this, usually 20 mini- tablets are selected randomly from each batch and their initial weight (W0) is noted. The mini-tablets were initially weighed and transferred into friabilator. The drum was rotated at 25 rpm for 4 minutes after which the mini-tablets were removed. Any loose dust was removed from the minitablets as before and the tablets were weighed again (Wfinal) ${ }^{39}$.

\section{Drug content uniformity}

Five mini-tablets weighted and crushed in a mortar then weighed powder contained equivalent to $10 \mathrm{mg}$ of drug transferred in $100 \mathrm{ml}$ of dissolution medium to give a concentration of $100 \mu \mathrm{g} / \mathrm{ml}$. Take $15 \mathrm{ml}$ of this solution and diluted it up to $100 \mathrm{ml}$ with same solution to give a concentration of $15 \mu \mathrm{g} / \mathrm{ml}$. Absorbance measured at respective wave length using UV-Visible spectrophotometer $^{40}$.

Table 1: List of mini tablets Available in the market

\begin{tabular}{|l|l|}
\hline Generic Name & Brand Name \\
\hline Donepezil Hydrochloride & Aricept \\
\hline Levonorgestrel and Ethinyl Estradiol & Alesse \\
\hline Sumatriptan and Naproxen Sodium Tablets & Treximet \\
\hline Pancrelipase & Ultresa \\
\hline Zafirlukast & Accolate \\
\hline Galantamine HBr ER & Razadyne ER \\
\hline Hydromorphone Hydrochloride Extended Release Tablets & Exalgo \\
\hline Warfarin Sodium & Coumadin \\
\hline Fenofibric Acid Capsules & Trilipix \\
\hline Vorapaxar Tablets & Zontivity \\
\hline Prasugrel Tablets & Effient \\
\hline Olanzapine & Zyprexa, Zyprexa Zydis \\
\hline
\end{tabular}

\section{In-vitro drug release}

Mini-tablets were subjected to in-vitro drug release studies in simulated gastric and intestinal fluids to assess their ability in providing the desired controlled drug delivery 35 . Drug release studies3,54, 55 were carried out using USP dissolution test apparatus I at $100 \mathrm{rpm}$, $37 \pm 0.5^{\circ} \mathrm{C}$, and $\mathrm{pH} 1.2$ buffer $(900 \mathrm{ml}$ ) (i.e. $0.1 \mathrm{~N} \mathrm{HCl}$ ) for 2 hours, since the average gastric emptying time is about 2 hours. The dissolution medium was replaced with $\mathrm{pH} 6.8$ phosphate buffer $(900 \mathrm{ml})$ and experiment continued for another 10 hours $^{36}$.At different time intervals, $5 \mathrm{ml}$ of the samples were withdrawn and replaced with $5 \mathrm{ml}$ of drugfree dissolution medium. The samples withdrawn were analyzed by UV spectrophotometer using multi component mode of analysis at required wave length ${ }^{37}$. In vitro drug release studies are carried out in USP type II dissolution test apparatus at specific rpm and temperature for definite time period in suitable buffer solution. All these factors depend on that particular formulation. From this, $10 \mathrm{ml}$ of sample is withdrawn and analyzed using UV spectrophotometer at appropriate wavelength. After this, drug release is tested for definite time period, at same temperature and same rotational speed. At all the time points $(15,30,60,90,120,240$ and 360 minutes $), 10 \mathrm{ml}$ of the sample is withdrawn, and analyzed using UVSpectrophotometer ${ }^{41}$.

\section{CONCLUSION}

From this review It can be easily concluded that Pharmaceutical mini tablet offer several advantages. Mini Tablets offer great advantage over single unit dosage forms. Accurate dose of drug can be given to patients to increase the efficiency. Compared to single unit dosage forms, mini tablets are good alternative to granules and pellets. However, production parameters must be carefully assessed to ensure a good flow, correct and complete filling of the die and damage to the equipment. Dose dumping and local irritation can be avoided by the use of mini tablets. For those drugs whose absorption is more in small intestine mini tablet dosage form is beneficial as they can easily pass through the duodenum independent of gastric emptying and intestinal motility.Bio adhesive mini tablets 
show increased bio adhesion and increased effect than that of single unit bio adhesive tablets. They are suitable for geriatric and pediatric patient groups compare to single

\section{REFERENCES}

[1] Banker G.S. and Anderson N.R., In; Lachman L., Liberman H.A. and Kanig J.L., Eds., The theory and practice of industrial pharmacy, IIIrd Edn., Lea and Febiger, Philadelphia, 1991, 293.

[2] Lennartz P., Mielc, J.B., Minitabletting: improving the compactability of paracetamol powder mixtures. Int. J. Pharm. 1998; 173:75-85. 8.

[3] Reddy L. H, Ghosh B, and Rajneesh. Fast dissolving drug delivery systems: a review of the literature, Indian Journal of Pharmaceutical Sciences, 2002; 64(4):331- 336

[4] Sastry SV, Nyshadham JR, Fix JA, Recent technological advances in oral drug delivery: a review, Pharm. Sci. Technol. Today 2000; 3(4):138-145

[5] Biradar SS, Bhagavaati ST, Kuppasad IJ, Fast dissolving drug delivery systems: a brief overview, Internet J. Pharmacol. 2006; $4: 2$.

[6] Lennartz, P., Mielck, J.B., Minitabletting: improving the compactability of paracetamol powder mixtures. Int. J. Pharm. 1998; 173:75-85r.

[7] ] Katakam VK, Reddy S, Panakanti PK, Yamsani MR, Design and Evaluation of a Novel Gas Formation-Based Multiple-Unit Gastro- Retentive Floating Drug Delivery System for Quetiapine Fumarate, Tropical Journal of Pharmaceutical Research April 2014; 13(4):489-496.

[8] Igwe J. Chibueze, Emenike IV, Oduola AR. Formulation and evaluation of Finasteride sustained-release matrix tablets using different rate controlling polymers. Univ J Pharm Res. 2016; $1(2): 25-31$

[9] Tawfeek HM, Saleem IY, Matthew Roberts, dissolution enhancement and formulation of rapid-release lornoxicam mini-tablets, pharmaceutics, drug delivery and pharmaceutical technology, 2470-2483.

[10] Tehseen N, Rao V, Hadi MA, Design and Characterization of Twice Daily Mini-tablets Formulation of Pregabalin, International Journal of Pharmacy and Pharmaceutical Sciences, 2013; 5(1):168-175.

[11] Mastoi SM, Ali A, Aslam H, Niaz K. Comparision of antidyslipiemic potential of 80 milligrams of Fenofibrated with 8 grams of Nigella sativa seeds daily. Univ J Pharm Res. 2017; 2(6):50-52.

[12] Mohamed FA, Roberts M, Seton L, Ford JL, Levina M, et al. Filmcoated matrix mini-tablets for the extended release of a watersoluble drug. Drug Dev. Ind. Pharm. 2015; 41:623-30.

[13] Keerthi ML, Kiran RS, Rao VUM, Sannapu A, Dutt AG, et al. Pharmaceutical Mini-Tablets, its Advantages, Formulation Possibilities and General Evaluation Aspects: A Review. Int. J. Pharm. Sci. Rev. Res. 2014; 28:214-221.

[14] ] Mahajan KV, Akarte1 AM, Sapate MK, Baviskar DT, Jain DK. Designing and evaluation of compressed mini-tablets of ramipril as a biphasic delivery system. Indo American Journal of Pharmaceutical Research. 2013; 3:7277-2787

[15] Lopes CM, Lobo JMS, Pinto JF, Costa P, Directly compressed mini matrix tablets containing ibuprofen: preparation and evaluation of sustained release. Drug. Dev. Ind. Pharm. 2006; 32:95-106.

[16] Pogula AH, Nazeer S, "Extended Release Formulation" International Journal Of Pharmacy \& Technology -2010.

[17] Modi SA, Gaikwad PD, Bankar VH, Pawar SP, "Sustained release drug delivery system: a review" International Journal of Pharma Research and Development., 2011; 11:147-198. 10 unit dose forms and also good substitutes for granules and pellets.

[18]Saddam C Shaikh, Dnyaneshwar S, Dipak V Bhusari, Jain S, Pooja P Kochar, Vikram N Sanchati. Formulation and evaluation of Ibuprofen gastro-retentive floating tablets. Univ J Pharm Res. 2018; 3(4):20-25.

[19] James Swarbick, Encyclopedia of Pharmaceutical Technology., 1993; 3: 2652-2663.

[20] Carla M. Lopes a, Jos'e Manuel Sousa Loboa, Jo ao F. Pinto b, Paulo Costa , Compressed mini-tablets as a biphasic delivery system, International Journal of Pharmaceutics 2006; 323:93100.

[21] Raghavendra Rao NG, Mohd Abdul Hadi, Harsh Panchal, A Novel approach to sustained Montelukast sodium release: Differentially coated mini-tablets in HPMC capsules, International Journal of Pharmaceutical and Biomedical Sciences, 2011; 2(2):90-97.

[22] Singh S, Virmani T, Virmani R, Kumar P, Mahlawat G. Fast dissolving drug delivery systems: formulation, preparation techniques and evaluation. Univ J Pharm Res. 2018; 3(4):60-69.

[23] Thomson SA, Tuleu C, Wong IC, Keady S, Pitt KG, Sutcliffe AG, Mini tablets: new modality to deliver medicines to preschoolaged children, Official Journal of the American Academy of Pediatrics, 2009; 123:e235-e238.

[24] Opeyemi OT, Adegbenro 00. Development and characterization of direct compressed matrix mini tablets of naproxen sodium. Universal Journal of Pharmaceutical Research. 2018; 3(5):7-11.

[25] Karthikeyan D, Vijayalaxmi A, Santhosh Kumar C, Formulation and evaluation of biphasic Delivery system of Aceclofenac minitablets in Hard gelatin capsules, International journal of novel trends in pharmaceutical sciences, 2013; 3(2):39-45.

[26] Solanki B, Patel R, Barot B, Parejiya P, Shelat P, Multiple Unit Dosage Forms: A Review, Pharmtechmedica, 2012; 1(1):11-21.

[27] Nweje-Anyalowu Paul C, Anyalogbu Ernest AA, White Alalibo Jim. Design and evaluation of chronotherapeutic pulsatile drug delivery system of Cilnidipine. Univ J Pharm Res. 2017; 2(5):1822.

[28] Mirelabodea, Ioan tomuţă, Sorin leucuţa, Identification of Critical Formulation Variables for Obtaining Metoprolol Tartrate Mini-tablets, Farmacia, 2010; 58:719-727.

[29] Chauhan V, Kumar K, Teotia D. Fast dissolving tablets: a promising approach for drug delivery. Univ J Pharm Res. 2017; 2(4):58-64.

[30] Garg D, Saini V, gupta S, Kapoor DN, joshi L. Controlled-release multiple-units and single-unit doses a literature review. Dhr international journal of pharmaceutical sciences 2013; 4(2):6673.

[31] Robert S. Rosenson Low high-density lipoprotein cholesterol and cardiovascular disease: Risk reduction with statin therapy Review Article American Heart Journal., 2006; 151(3):556-56.

[32] Tong Zhu, Jean-Claude Ansquer, Maureen T. Kelly, Darryl J. Sleep, Rajendra S. Pradhan, Comparison of the Gastrointestinal Absorption and Bioavailability of Fenofibrate and Fenofibric Acid in Humans, Journal Of Clinical Pharmacology., 2010; 50:914-921.

[33] Chen, Yisheng Qiu, Yihong Reiland, ThomasL, "Pharmaceutical Compositions Comprising Effervescent Agents and Fenofibrate" United States Patent Application 20100021393. 
[34] Abdulmajed A, Alyahawi A. Quality assessment of different brands of paracetamol tablets in Yemeni market. Univ J Pharm Res. 2018; 3(4):42-47.

[35] Vogt M, Kunath K, "Dissolution enhancement of fenofibrate by micronization, cogrinding and spray-drying: Comparison with commercial preparations" European Journal of PharmaceuticsandBiopharmaceutics., 2008; 68:283-288.

[36] Gundu, Ramakant kashinath (Maharashtra) Murali, Narayanan (tamilnadu, in) Jain, Girish kumar (dehli) "Pharmaceutical compositions of fenofibrate" United States Patent Application 20100255095.

[37] Srinarong P, Faber JH, "Strongly enhanced dissolution rate of fenofibrate solid dispersion tablets by incorporation of superdisintegrants" European Journal of Pharmaceutics and Biopharmaceutics., 2009; 154-161.
[38] Zhu Z, Zheng L. Development and mathematical simulation of theophylline pulsatile release tablets. Drug Dev Ind Pharm. 2005; 31:1009-1017. doi: 10.1080/03639040500306278.

[39] Smolenska Z, Kaznowska Z, Zarowny D, Simmonds HA, Smolenski RT. Effect of methotrexate on blood purine and pyrimidine levels in patients with rheumatoid arthritis. Rheumatology (Oxford) 1999; 38:997-1002. doi: 10.1093/rheumatology/38.10.997.

[40] Tehseen N, Rao V, Hadi MA, Design and Characterization of Twice Daily Mini-tablets Formulation of Pregabalin, International Journal of Pharmacy and Pharmaceutical Sciences, 2013; 5(1):168-175.

[41] Alyahawi A, Abdulmajed A. Quality control assessment of different brands of ciprofloxacin $500 \mathrm{mg}$ tablets in Yemen. Univ J Pharm Res. 2018; 3(4):31-36. 